Supporting Information

For

\title{
Reversible Photoregulation of $\alpha$-Chymotrypsin Binding to a Gold Surface
}

David Pearson, Alison J. Downard, Andrew Muscroft-Taylor, and Andrew D.

$$
\text { Abell }{ }^{\star, *}
$$

Contribution from the Department of Chemistry, University of Canterbury,

Christchurch, New Zealand

$\$$ Current address: School of Chemistry and Physics, University of Adelaide, SA 5005, Australia

\section{Contents of supporting information:}

1) Experimental procedures

Page S2

2) Calculation of the maximum possible SPR response on binding of $\alpha$-chymotrypsin to the modified surface

Page S5

3) Synthesis of compounds $3,5,6,8$ and 1

Page S6

4) ${ }^{1} \mathrm{H}$ and ${ }^{13} \mathrm{C}$ NMR spectra for compounds $3,5, \mathbf{6 ,}, \mathbf{8}$ and $\mathbf{1}$

Page S9

5) References

Page S14 


\section{1) Experimental procedures}

\section{General}

NMR spectra were obtained on a Varian INOVA spectrometer, operating at $500 \mathrm{MHz}$ for ${ }^{1} \mathrm{H}$ NMR and at $126 \mathrm{MHz}$ for ${ }^{13} \mathrm{C} \mathrm{NMR}$, or on a Varian UNITY 300 spectrometer, operating at $75 \mathrm{MHz}$ for ${ }^{13} \mathrm{C}$ NMR. Two-dimensional NMR experiments including COSY and HSQC were used to assign spectra, and were obtained on the Varian INOVA spectrometer operating at $500 \mathrm{MHz}$. Electrospray ionisation mass spectra were detected on a Micromass LCT TOF mass spectrometer operating in electrospray mode with $1: 1$ acetonitrile/ $\mathrm{H}_{2} \mathrm{O}$ as solvent. Dry DMF was purchased from Acros, HPLC grade acetonitrile was purchased from BDH. Dry THF was distilled from sodium or potassium, dry DCM and EtOAc were distilled from $\mathrm{CaH}_{2}$. Milli-Q water was used for all assays and SPR experiments including surface preparation.

\section{Solution photoisomerisation}

Compound 1 was photoisomerized by irradiation with UV or visible light from a $500 \mathrm{~W}$ mercury arc lamp. The light beam was filtered through water to reduce heat, and through either a Corning 7 37 filter for UV, or a Corning 0-51 visible filter for visible light. A sample of 1 (2 mg) was dissolved in $\mathrm{d}_{3}$-acetonitrile $(600 \mu \mathrm{L})$ and irradiated for $90 \mathrm{~min}$ with UV light, or for 30 min with visible light. The ratio of $E: Z$ isomers was determined before and after irradiations by ${ }^{1} \mathrm{H}$ NMR analysis using the integrals of well separated peaks. The major isomer was assigned the thermodynamically more stable $E$ configuration based on literature precedence. ${ }^{1,2}$ The minor $Z$ isomer characteristically gave rise to upfield signals for the aryl protons which were enhanced on UV irradiation. All experiments involving photoisomerisation were carried out in dim lighting conditions, i.e. glassware wrapped in foil and with the lights turned off.

\section{Solution enzyme assay}

Buffer solution (Tris): Tris(hydroxymethyl)aminomethane (1.21 g), $\mathrm{CaCl}_{2} \cdot 6 \mathrm{H}_{2} \mathrm{O}(0.44 \mathrm{~g})$ and Triton $\mathrm{X}-100(0.05 \mathrm{~g})$ were dissolved in water $(75 \mathrm{~mL})$, adjusted to $\mathrm{pH} 7.8$ with $1 \mathrm{M} \mathrm{HCl}$ solution and made up to $100 \mathrm{~mL}$ with water. 
Substrate solution: N-Succinyl-(Ala) $)_{2}$-Pro-Phe-4-nitroanilide $(21 \mathrm{mg})$ was dissolved in Tris buffer solution $(10 \mathrm{~mL})$ by ultrasonication. The concentration of the solution was determined from its UV spectrum $\left(\varepsilon 315=14000 \mathrm{Lmol}^{-1} \mathrm{~cm}^{-1}\right)$

Enzyme solution: A stock solution was prepared from $\alpha$-chymotrypsin (15 mg) in $\mathrm{HCl}$ solution (10 $\mathrm{mL}, \mathrm{pH} 3$, made up by dilution of conc. $\mathrm{HCl}$ with Milli-Q water). An enzyme solution was prepared for assay by dilution of the stock solution $(100 \mu \mathrm{L})$ and Triton X-100 $(25 \mathrm{mg})$ to $50 \mathrm{~mL}$ with water.

Inhibition of $\alpha$-chymotrypsin was determined with Suc-Ala-Ala-Pro-Phe-4-nitroanilide as the substrate by an assay procedure developed from the technique described by Geiger, ${ }^{3}$ except that the order of addition of enzyme and substrate was inverted, and only one substrate concentration was used, in order to obtain inhibition constants as $\mathrm{IC}_{50}$ rather than $\mathrm{Ki}$. Briefly, $\mathbf{1}$ was dissolved in $\mathrm{d}_{3^{-}}$ acetonitrile at a series of dilutions: 3, 6, 13, 26 and $51 \mu \mathrm{M}$. For each rate measurement, inhibitor solution (or acetonitrile blank, $50 \mu \mathrm{L})$, substrate solution $(60 \mu \mathrm{L})$ and buffer solution $(910 \mu \mathrm{L})$ were mixed in a cuvette, and incubated for $5 \mathrm{~min}$ at $25^{\circ} \mathrm{C}$. Enzyme solution $(30 \mu \mathrm{L})$ was added, and the absorbance at $405 \mathrm{~nm}$ was monitored for $10 \mathrm{~min}$. An absorbance vs time plot was obtained, and the final slope used to find the rate of reaction. From the difference between the rate and the rate for the acetonitrile blank, the percent inhibition was calculated. This experiment was repeated for each inhibitor concentration, and the $\mathrm{IC}_{50}$ was interpolated from a plot of percent inhibition vs $\log [$ inhibitor concentration].

\section{Surface Plasmon Resonance experiments}

SPR was performed using a Biacore 2000 instrument. All solutions were filtered through a $0.22 \mu \mathrm{M}$ filter and centrifuged (1300 rpm, $10 \mathrm{sec})$ before use.

Buffers used: HEPES (0.01 M, pH 7.4 containing $0.15 \mathrm{M} \mathrm{NaCl}$ and $3 \mathrm{mM}$ EDTA), Tris (0.1 M, pH 7.8, containing $0.02 \mathrm{M} \mathrm{CaCl}_{2}$ and $12.5 \mu \mathrm{L} \mathrm{P} 20$ surfactant).

Compound 1 was immobilised to an SPR chip as follows: A Biacore CM5 chip was docked in the SPR instrument and prepared by priming and running a sensorgram in HEPES buffer at $100 \mu \mathrm{L} / \mathrm{min}$ flow rate, injecting $\mathrm{NaOH}(50 \mathrm{mM}, 20 \mu \mathrm{L} \times 2), \mathrm{HCl}(10 \mathrm{mM}, 20 \mu \mathrm{L} \times 2)$, $\operatorname{SDS}(0.1 \%, 20 \mu \mathrm{L} \times 2)$. Surface modification was then carried out at $5 \mu \mathrm{L} / \mathrm{min}$ flow rate by injection of $35 \mu \mathrm{L}$ EDCI/NHS, 
followed by $9(11 \mathrm{mg} / \mathrm{mL}$ in HEPES buffer, $3 \times 5 \mu \mathrm{L})$ then ethanolamine $(1 \mathrm{M}, \mathrm{pH} 8.5,35 \mu \mathrm{L})$. During attachment of $\mathbf{9}$, the SPR resonance increased by 147 RU. The chip was then undocked and removed from the instrument and rinsed with water. A solution of $1(0.3 \mathrm{mg}, 0.44 \mu \mathrm{mol}), \mathrm{CuBr}$ (0.063 mg, $0.44 \mu \mathrm{mol}), 2,6$-lutidine $(0.094 \mathrm{mg}, 0.88 \mu \mathrm{mol}), 2,2$ '-bipyridine $(0.14 \mathrm{mg}, 0.88 \mu \mathrm{mol})$, sodium ascorbate $(0.17 \mathrm{mg}, 0.88 \mu \mathrm{mol})$ in $1: 1 \mathrm{DMF} / \mathrm{H}_{2} \mathrm{O}(150 \mu \mathrm{L})$ was pipetted onto the surface and left for $1 \mathrm{~h}$ at room temperature. The surface was then rinsed with 1:1 DMF/ $\mathrm{H}_{2} \mathrm{O}, \mathrm{H}_{2} \mathrm{O}, 0.1 \mathrm{M}$ EDTA, 1:1 DMF/ $\mathrm{H}_{2} \mathrm{O}, \mathrm{H}_{2} \mathrm{O}$ then redocked in the SPR instrument. A sensorgram was run in Tris buffer for several hours in order to obtain a stable baseline. A reference cell was prepared using the same method except omitting the injection of $\mathbf{9}$.

Enzyme binding to this modified surface was monitored by running a sensorgram in tris buffer at 30 $\mu \mathrm{L} / \mathrm{min}$. A range of $\alpha$-chymotrypsin solution concentrations were injected over the surface and monitored as follows:

An $\alpha$-chymotrypsin solution (either $0,2.0,6.0$ or $18 \mu \mathrm{M}$ in Tris buffer, $150 \mu \mathrm{L}$ ) was injected over a period of $5 \mathrm{~min}$, flow rate $30 \mu \mathrm{L} / \mathrm{min}$. After the injection was complete, buffer was run for $5 \mathrm{~min}$ to monitor dissociation of enzyme from the surface, then the surface was regenerated. Regeneration was carried out at $5 \mu \mathrm{L} / \mathrm{min}$ in Tris buffer by the injection of guanidine hydrochloride $(6 \mathrm{M}, 5 \mu \mathrm{L})$ followed by acetic acid $(1 \mathrm{M}, 10 \mu \mathrm{L})$, then buffer was run for $5 \mathrm{~min}$.

For photoswitching experiments, the above enzyme binding assay was carried out, then the chip was regenerated, removed from the instrument, rinsed with water and irradiated with UV light (as detailed above for solution-phase photoisomerisation) for $10 \mathrm{~min}$. The chip was then replaced in the SPR instrument, and the enzyme binding assay was repeated, using only $2.0 \mu \mathrm{M}$ enzyme solution. The photoisomerisation process was repeated with visible light irradiation and this assay was repeated. 
2) Calculation of the maximum possible SPR response on binding of $\alpha$-chymotrypsin to the modified surface

Assuming that every azide group at the surface reacts with a molecule of alkyne $\mathbf{1}$ during the immobilisation, the amount of azide 9 (147 Resonance Units (RU)) that was attached to the surface can be used to estimate the maximum enzyme binding capacity of this surface (in RU) as follows (see Biacore Sensor Surface Handbook section 3.6.1):

Maximum binding capacity $(\mathrm{RU})=$ MW (chymotrypsin) $\quad \times \quad$ Immobilised $9(\mathrm{RU})$

MW (azide 9)

$$
\begin{aligned}
& =\quad 25000 / 218 \times 147 \\
& =16838
\end{aligned}
$$

Thus, the maximum possible response that can be obtained on chymotrypsin binding to this surface is $17000 \mathrm{RU}$. 


\section{3) Synthesis of compounds 3, 5, 6, 8 and 1}

2,2-Dimethyl-6,9,12,15,18-pentaoxa-2-silahenicosa-3,20-diyne (3). To a solution of $\mathbf{2}^{4}$ ( $2.1 \mathrm{~g}, 7.7$ $\mathrm{mmol})$ in THF $(25 \mathrm{~mL})$ at $-78^{\circ} \mathrm{C}$ was added $n$-BuLi $(5.3 \mathrm{~mL}$ of $1.6 \mathrm{M}$ solution in hexanes, 8.5 mmol) dropwise. The mixture was stirred at $-78^{\circ} \mathrm{C}$ for $1 \mathrm{~h}$ then TMS-Cl $(1.1 \mathrm{~mL}, 8.6 \mathrm{mmol})$ was added dropwise. The solution was stirred at $-78^{\circ} \mathrm{C}$ for $1 \mathrm{~h}$ then allowed to warm to room temperature and stirred for $16 \mathrm{~h}$. The solution was concentrated, and the crude material purified by flash chromatography, eluting with a gradient of EtOAc to petroleum ether to give $3(0.68 \mathrm{~g}, 26 \%)$ and $2(0.95 \mathrm{~g}, 46 \%)$ as colourless oils: $\mathrm{Rf}=0.39$ (1:1 EtOAc:petroleum ether), IR $\left(\mathrm{KBr}, \mathrm{cm}^{-1}\right)$ : 2957, 2870, 2361, 2341, 1445, 1350, 1252, 1103, 1034, 991, 845, ${ }^{1} \mathrm{H}$ NMR (500MHz, $\left.\mathrm{CDCl}_{3}\right): \delta$ $0.03(9 \mathrm{H}, \mathrm{s}), 2.35(1 \mathrm{H}, \mathrm{t}, \mathrm{J}=2.4 \mathrm{~Hz}), 3.51(8 \mathrm{H}, \mathrm{s}), 3.53(6 \mathrm{H}, \mathrm{s}), 3.52-3.56(2 \mathrm{H}, \mathrm{m}), 4.05(2 \mathrm{H}, \mathrm{d}, J=$ $1.2 \mathrm{~Hz}), 4.06(2 \mathrm{H}, \mathrm{d}, \mathrm{J}=1.1 \mathrm{~Hz}),{ }^{13} \mathrm{C} \mathrm{NMR}\left(75 \mathrm{MHz}, \mathrm{CDCl}_{3}\right): \delta-0.5,58.0,58.7,68.6,68.7,70.0$, 70.0, 70.2, 70.2, 70.2, 70.2, 74.3, 79.3, 90.0, 100.2. $\mathrm{m} / \mathrm{z}$ (ES) 343.1938, calcd for $\mathrm{C}_{17} \mathrm{H}_{31} \mathrm{O}_{5} \mathrm{Si}$ $\left(\mathrm{MH}^{+}\right) 343.1941$.

\section{(E)-methyl 4-((4-(2,2-dimethyl-6,9,12,15,18-pentaoxa-2-silahenicosa-3,20-diyn-21-yl)phenyl)}

diazenyl)benzoate (5). The aryl iodide $4^{5}(260 \mathrm{mg}, 0.70 \mathrm{mmol}), 3(200 \mathrm{mg}, 0.58 \mathrm{mmol})$ and $\mathrm{Pd}\left(\mathrm{PPh}_{3}\right)_{2} \mathrm{Cl}_{2}(21 \mathrm{mg}, 5 \mathrm{~mol} \%)$ were evacuated at $0.1 \mathrm{~mm} \mathrm{Hg}$ then placed under an Ar atmosphere. DMF $(15 \mathrm{~mL})$ and $\mathrm{NEt}_{3}(7.5 \mathrm{~mL})$ were added and the resultant solution was heated at $55^{\circ} \mathrm{C}$ for $12 \mathrm{~h}$. The solution was concentrated, then the crude material was poured into aqueous $\mathrm{HCl}$ solution (1 M, $100 \mathrm{~mL})$ and extracted with $\mathrm{CH}_{2} \mathrm{Cl}_{2}(20 \mathrm{~mL} \times 3)$. The combined organic fractions were washed with $\mathrm{H}_{2} \mathrm{O}(50 \mathrm{~mL})$, brine $(50 \mathrm{~mL})$, dried $\left(\mathrm{Na}_{2} \mathrm{SO}_{4}\right)$ and concentrated. The crude material was purified by flash chromatography, eluting with $2: 3 \mathrm{EtOAc} /$ petroleum ether to give 5 (170 $\mathrm{mg}$, 49\%): Rf: 0.45 (1:1 EtOAc/petroleum ether), IR ( $\left.\mathrm{KBr}, \mathrm{cm}^{-1}\right): 2870,1720,1437,1348,1281,1252$, 1103, 849, 775, 760, ${ }^{1} \mathrm{H}$ NMR (500MHz, $\left.\mathrm{CDCl}_{3}\right): \delta 0.17(9 \mathrm{H}, \mathrm{s}), 3.66-3.70(12 \mathrm{H}, \mathrm{m}), 3.76$ (4H, ddd, $J=3.9,6.5,9.0 \mathrm{~Hz}), 3.96(3 \mathrm{H}, \mathrm{s}), 4.20(2 \mathrm{H}, \mathrm{s}), 4.47(2 \mathrm{H}, \mathrm{s}), 7.60(2 \mathrm{H}, \mathrm{dt}, \mathrm{J}=1.9,8.6 \mathrm{~Hz}), 7.91(2 \mathrm{H}$, $\mathrm{dt}, J=19,8.6 \mathrm{~Hz}), 7.95(2 \mathrm{H}, \mathrm{dt}, \mathrm{J}=1.8,8.7 \mathrm{~Hz}), 8.20(2 \mathrm{H}, \mathrm{dt}, J=1.8,8.7 \mathrm{~Hz}),{ }^{13} \mathrm{C} \mathrm{NMR}(75 \mathrm{MHz}$, $\left.\mathrm{CDCl}_{3}\right): \delta 52.2,59.0,59.0,68.9,69.1,70.2,70.3,70.4,70.4,70.4,70.5,85.7,88.0,91.2,101.3$, $122.5,122.9,125.9,130.4,131.8,132.4,151.5,154.8,166.2, \mathrm{~m} / \mathrm{z}$ (ES) 581.2657 , calcd for $\mathrm{C}_{31} \mathrm{H}_{41} \mathrm{~N}_{2} \mathrm{O}_{7} \mathrm{Si}\left(\mathrm{MH}^{+}\right)$581.2683.

(E)-4-((4-(4,7,10,13,16-pentaoxanonadeca-1,18-diynyl)phenyl)diazenyl)benzoic acid (6). To a solution of $5(50.0 \mathrm{mg}, 0.096 \mathrm{mmol})$ in $2: 1 \mathrm{THF} / \mathrm{MeOH}(3 \mathrm{~mL})$ at room temperature was added a solution of aqueous $\mathrm{NaOH}(0.8 \mathrm{M}, 1.5 \mathrm{~mL})$. The resulting mixture was stirred for $14 \mathrm{~h}$, then diluted with aqueous $\mathrm{HCl}(1 \mathrm{M}, 5 \mathrm{~mL})$ and extracted with $\mathrm{Et}_{2} \mathrm{O}(5 \mathrm{~mL} \times 3)$. The combined organic fractions 
were washed with $\mathrm{H}_{2} \mathrm{O}(5 \mathrm{~mL})$, brine $(5 \mathrm{~mL})$, dried $\left(\mathrm{Na}_{2} \mathrm{SO}_{4}\right)$ and concentrated. The crude material was purified by flash chromatography eluting with 1:1 EtOAc/petroleum ether to give 6 as an orange oil (27 mg, 64\%): Rf: 0.46 (3:1 EtOAc/petroleum ether), IR (KBr, cm $\left.{ }^{-1}\right): 2864,2557,1680$, 1425, 1350, 1294, 1103, 866, 841, ' $\mathrm{H}$ NMR (500MHz, $\left.\mathrm{CDCl}_{3}\right): \delta 2.42(1 \mathrm{H}, \mathrm{t}, \mathrm{J}=2.4 \mathrm{~Hz}), 3.66-$ $3.68(4 \mathrm{H}, \mathrm{m}), 3.68-3.71(4 \mathrm{H}, \mathrm{m}), 3.73-3.75(2 \mathrm{H}, \mathrm{m}), 3.79-3.81(2 \mathrm{H}, \mathrm{m}), 4.21(2 \mathrm{H}, \mathrm{d}, \mathrm{J}=2.4 \mathrm{~Hz})$, $4.48(2 \mathrm{H}, \mathrm{s}), 7.60(2 \mathrm{H}, \mathrm{d}, \mathrm{J}=8.6 \mathrm{~Hz}), 7.91(2 \mathrm{H}, \mathrm{d}, \mathrm{J}=8.6 \mathrm{~Hz}), 7.98(2 \mathrm{H}, \mathrm{d}, \mathrm{J}=8.7 \mathrm{~Hz}), 8.26(2 \mathrm{H}$, $\mathrm{d}, \mathrm{J}=8.7 \mathrm{~Hz}),{ }^{13} \mathrm{C} \mathrm{NMR}\left(75 \mathrm{MHz}, \mathrm{CDCl}_{3}\right): \delta 58.3,59.1,69.0,69.2,70.3,70.3,70.4,70.4,70.5$, $74.5,79.5,85.8,88.0,122.7,123.0,126.0,131.1,131.4,132.5,151.6,155.2,170.0, \mathrm{~m} / \mathrm{z}(\mathrm{ES})$ 495.2118, calcd for $\mathrm{C}_{27} \mathrm{H}_{31} \mathrm{~N}_{2} \mathrm{O}_{7}\left(\mathrm{MH}^{+}\right) 495.2131$.

\section{(3S,E)-methyl 3-(4-((4-(4,7,10,13,16-pentaoxanonadeca-1,18-diynyl)phenyl)diazenyl)}

benzamido)-2-hydroxy-4-phenylbutanoate (8). To a solution of 6 (100 mg, $0.20 \mathrm{mmol}), 7^{6}$ (44 mg, 1 eq), EDCI (43 mg, 1.1 eq) and HOBt (30 mg, 1.1 eq) in DMF (5 mL) was added DIEA (77 $\mathrm{mL}, 57 \mathrm{mg}, 2.2 \mathrm{eq})$ and the resulting mixture was stirred for $19 \mathrm{~h}$, diluted with EtOAc $(50 \mathrm{~mL})$, washed with $\mathrm{H}_{2} \mathrm{O}(50 \mathrm{~mL} \times 4)$, brine $(50 \mathrm{~mL})$, dried over $\mathrm{MgSO}_{4}$ and concentrated. The crude product was purified by column chromatography, eluting with 2:3 EtOAc/DCM to give 8 (110 mg, $80 \%)$ as an orange gel: ${ }^{1} \mathrm{H} \mathrm{NMR}\left(500 \mathrm{MHz}, \mathrm{CDCl}_{3}\right) \delta 2.54\left(1 \mathrm{H}, \mathrm{s}, \mathrm{OCH}_{2} \mathrm{CCH}\right), 3.00-3.20(2 \mathrm{H}, \mathrm{m}$, $\left.\mathrm{CHCH}_{2}\right), 3.55-3.80(16 \mathrm{H}, \mathrm{m}), 4.18(1 \mathrm{H}, \mathrm{t}, \mathrm{J}=6.1 \mathrm{~Hz}), 4.24\left(2 \mathrm{H}, \mathrm{d}, \mathrm{J}=2.2 \mathrm{~Hz}, \mathrm{CHCCH}_{2}\right), 4.52(2 \mathrm{H}$, s), $4.75(1 \mathrm{H}, \mathrm{m}, \mathrm{NHCH}), 6.18(1 \mathrm{H}, \mathrm{d}, \mathrm{J}=7.0 \mathrm{~Hz}), 7.24-7.43(5 \mathrm{H}, \mathrm{m}), 7.50(1 \mathrm{H}, \mathrm{d}, \mathrm{J}=8.3 \mathrm{~Hz}), 7.61$ $(2 \mathrm{H}, \mathrm{d}, \mathrm{J}=8.4 \mathrm{~Hz}), 7.85-7.95(6 \mathrm{H}, \mathrm{m}){ }^{13} \mathrm{C} \mathrm{NMR}\left(126 \mathrm{MHz}, \mathrm{CDCl}_{3}\right) \delta 37.4\left(\mathrm{CHCH}_{2}\right), 50.8(\mathrm{NHCH})$, 58.1, 58.9, 68.7, 69.0, 70.0, 70.1, 70.2, 70.2, 70.3, 74.6, 79.3, 85.7, 87.7, 122.7, 122.8, 125.6, 126.7, $127.8,128.5,129.1,132.3,135.7,136.7,151.3,153.8,167.4, \mathrm{~m} / \mathrm{z}$ (ES) 718.2735 , calcd for $\mathrm{C}_{37} \mathrm{H}_{40} \mathrm{~F}_{3} \mathrm{~N}_{3} \mathrm{NaO}_{7}\left(\mathrm{MNa}^{+}\right) 718.2716$.

\section{(S,E)-methyl 3-(4-((4-(4,7,10,13,16-pentaoxanonadeca-1,18-diynyl)phenyl)diazenyl)}

benzamido)-2-oxo-4-phenylbutanoate (1). A solution of 8 (31 mg, $0.045 \mathrm{mmol}$ ) and Dess-Martin periodinane $(70 \mathrm{mg}, 3.7 \mathrm{eq})$ in DCM $(2 \mathrm{~mL})$ was stirred for $3 \mathrm{~h}$, diluted with EtOAc $(50 \mathrm{~mL})$, washed with $\mathrm{Na}_{2} \mathrm{~S}_{2} \mathrm{O}_{3}\left(0.25 \mathrm{M}\right.$ in sat. $\left.\mathrm{NaHCO}_{3}, 50 \mathrm{~mL} \times 2\right)$, sat. $\mathrm{NaHCO}_{3}(50 \mathrm{~mL} \times 2)$, brine $(50$ $\mathrm{mL}$ ), dried over $\mathrm{MgSO}_{4}$ and concentrated. The crude material was purified by column chromatography, eluting with 1:1 EtOAc/DCM to give $3.88 .1(28 \mathrm{mg}, 90 \%)$ as an orange gel: ${ }^{1} \mathrm{H}$ NMR $\left(500 \mathrm{MHz},\left(\mathrm{CD}_{3}\right)_{2} \mathrm{CO}\right) \delta 2.92\left(1 \mathrm{H}, \mathrm{t}, \mathrm{J}=2.4 \mathrm{~Hz}, \mathrm{OCH}_{2} \mathrm{CCH}\right), 3.25(1 \mathrm{H}, \mathrm{dd}, \mathrm{J}=12.1,14.0 \mathrm{~Hz}$, $\left.\mathrm{CHCH}_{2}\right), 3.40\left(1 \mathrm{H}, \mathrm{dd}, \mathrm{J}=3.1,14.0 \mathrm{~Hz}, \mathrm{CHCH}_{2}\right), 3.55-3.65(12, \mathrm{~m}), 3.67(2 \mathrm{H}, \mathrm{m}), 3.74(2 \mathrm{H}, \mathrm{m})$, $4.17\left(2 \mathrm{H}, \mathrm{d}, \mathrm{J}=2.4 \mathrm{~Hz}, \mathrm{CHCCH}_{2} \mathrm{O}\right), 4.40-4.48(3 \mathrm{H}, \mathrm{m}), 6.80(1 \mathrm{H}, \mathrm{s}), 7.14(1 \mathrm{H}, \mathrm{s}), 7.19(1 \mathrm{H}, \mathrm{t}, \mathrm{J}=$ $7.4 \mathrm{~Hz}), 7.27(2 \mathrm{H}, \mathrm{t}, \mathrm{J}=7.4 \mathrm{~Hz}), 7.34(2 \mathrm{H}, \mathrm{d}, \mathrm{J}=7.4 \mathrm{~Hz}), 7.67(2 \mathrm{H}, \mathrm{d}, \mathrm{J}=8.6), 7.88-7.94(6 \mathrm{H}, \mathrm{m})$, $8.20(1 \mathrm{H}, \mathrm{d}, \mathrm{J}=8.1 \mathrm{~Hz}){ }^{13} \mathrm{C} \mathrm{NMR}\left(126 \mathrm{MHz},\left(\mathrm{CD}_{3}\right)_{2} \mathrm{CO}\right) \delta 33.7\left(\mathrm{CHCH}_{2}\right), 58.5,58.5,59.3,69.7$, 
70.0, 70.9, 71.1, 71.2, 71.2, 75.7, 80.9, 85.8, 89.8, $95.3\left(\mathrm{q}, \mathrm{J}=29.8, \mathrm{CCF}_{3}\right), 123.4,123.9,127.0$, $127.2,129.2,129.5,130.1,133.5,137.0,139.5,152.6,155.0,169.6, \mathrm{~m} / \mathrm{z}$ (ES) 734.2652 , calcd for $\mathrm{C}_{37} \mathrm{H}_{40} \mathrm{~F}_{3} \mathrm{~N}_{3} \mathrm{NaO}_{8}\left(\mathrm{M}^{\left.-\mathrm{H}_{2} \mathrm{O} . \mathrm{Na}^{+}\right)}\right.$734.2665. 
4) ${ }^{1} \mathrm{H}$ and ${ }^{13} \mathrm{C}$ NMR spectra of compounds 3, 5, 6, 8 and 1

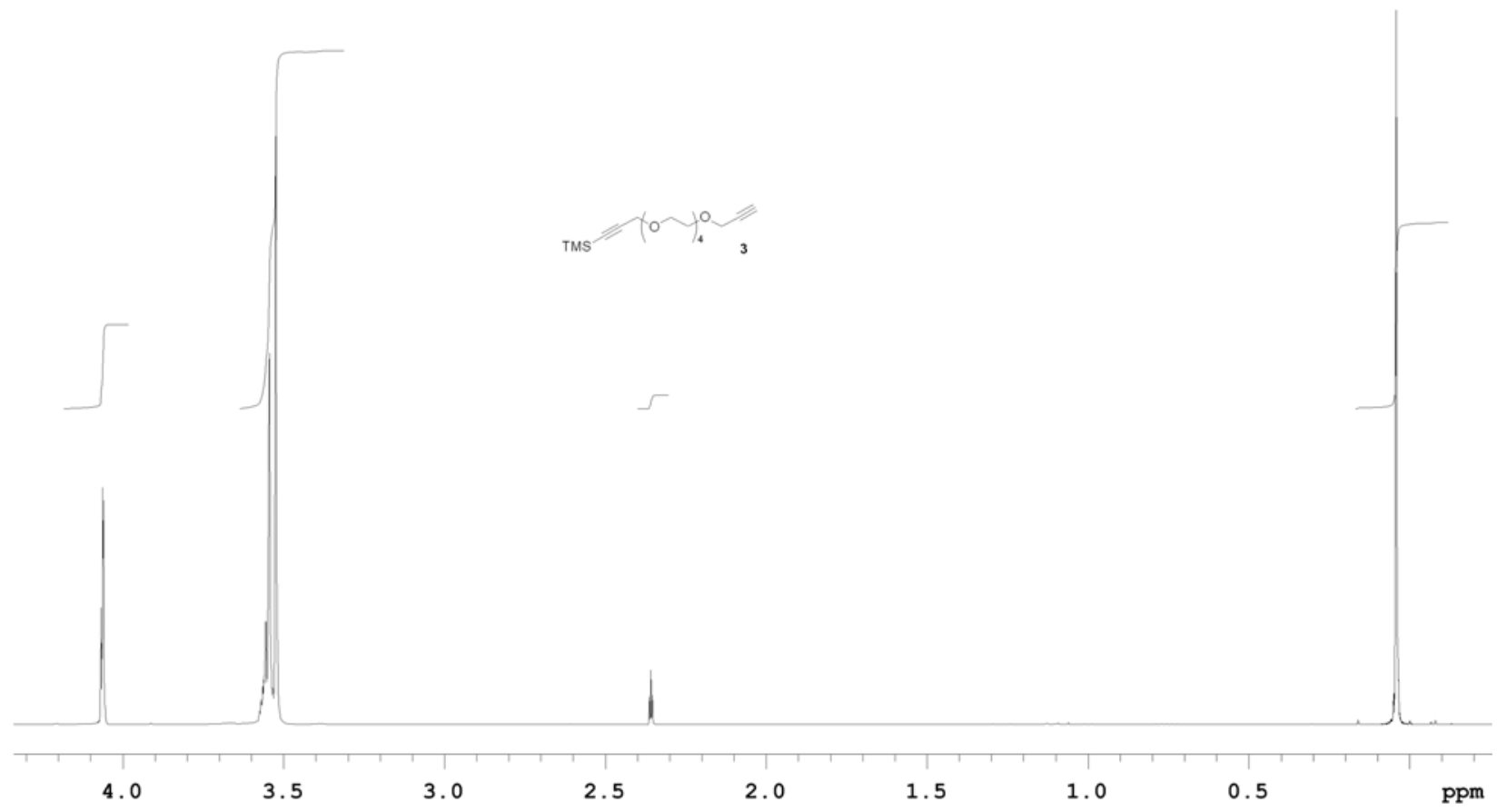

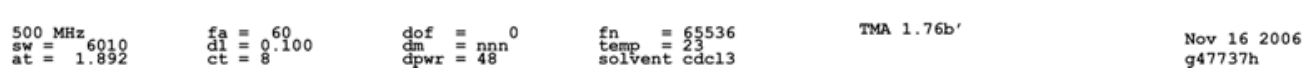

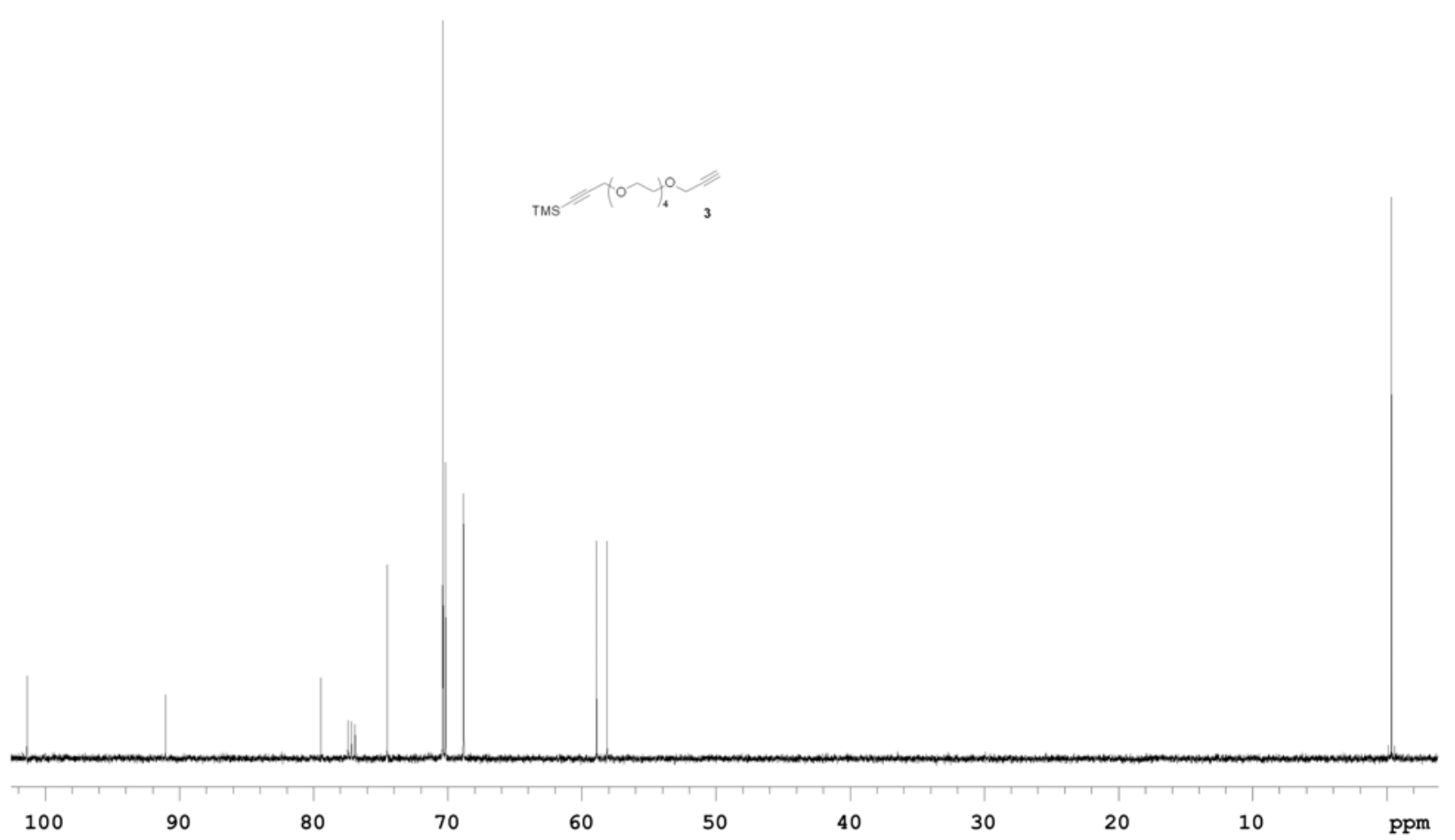

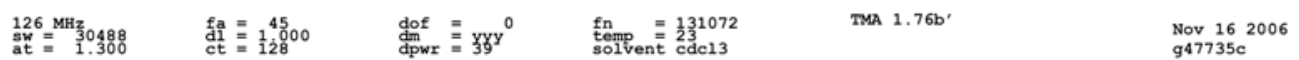



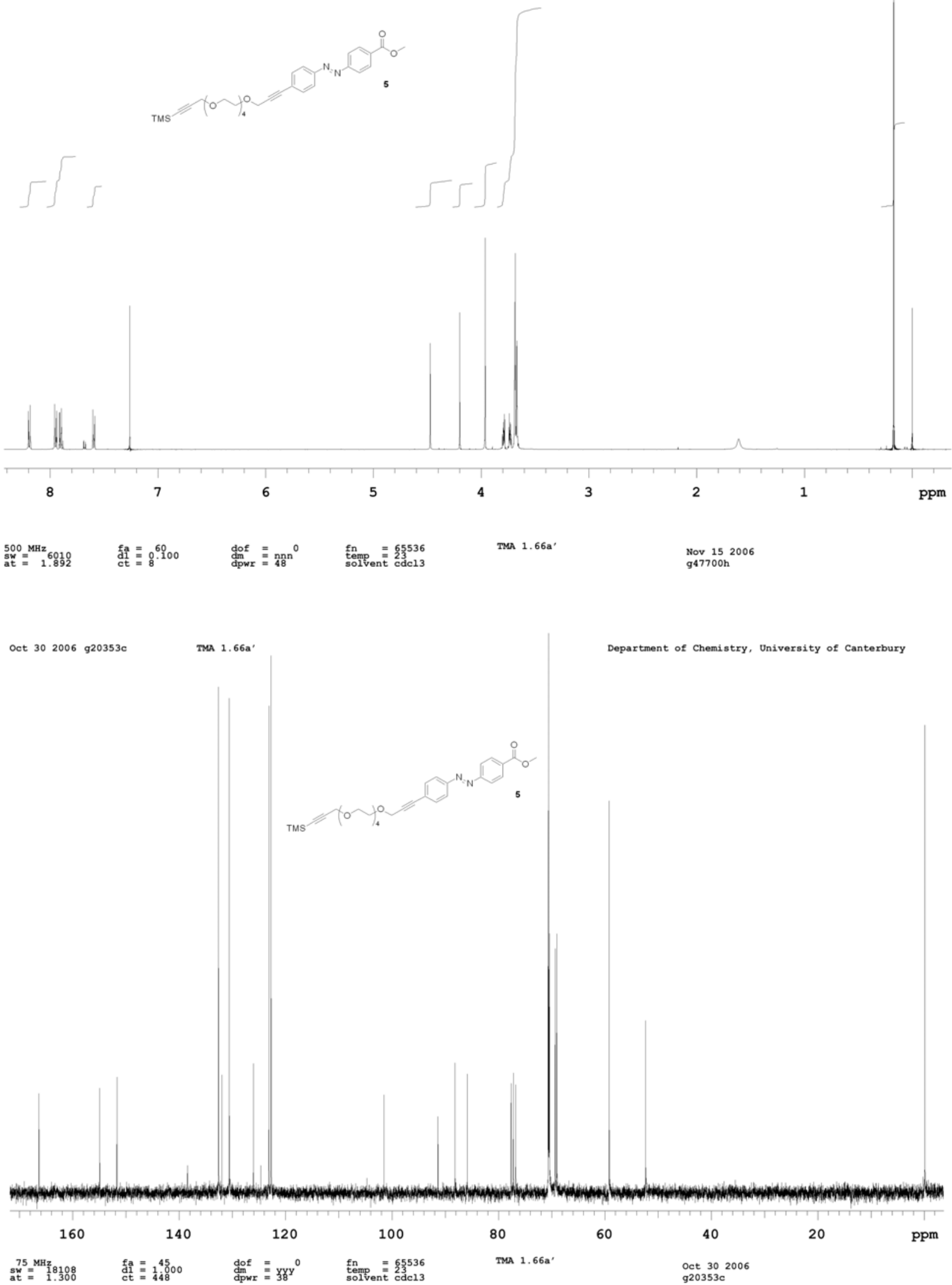


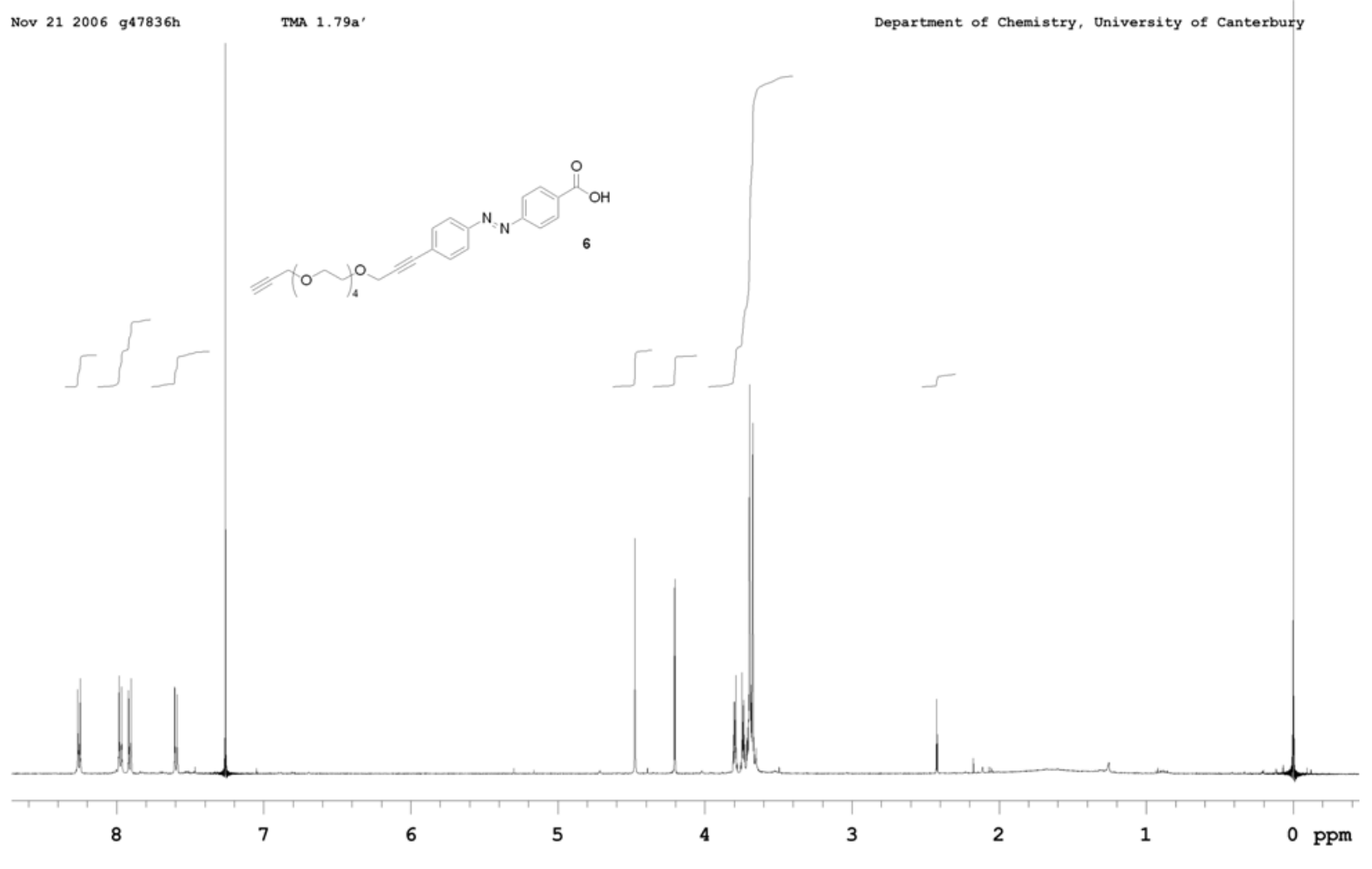

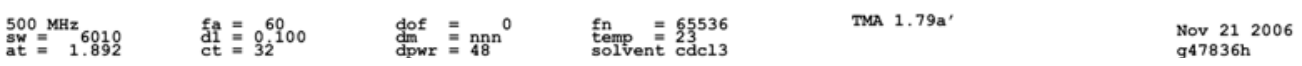

\begin{tabular}{l|l|l} 
Nov $212006 j 20461 \mathrm{c2} \quad$ TMA 1.79a' Department of Chemistry, University of Canterbury
\end{tabular}

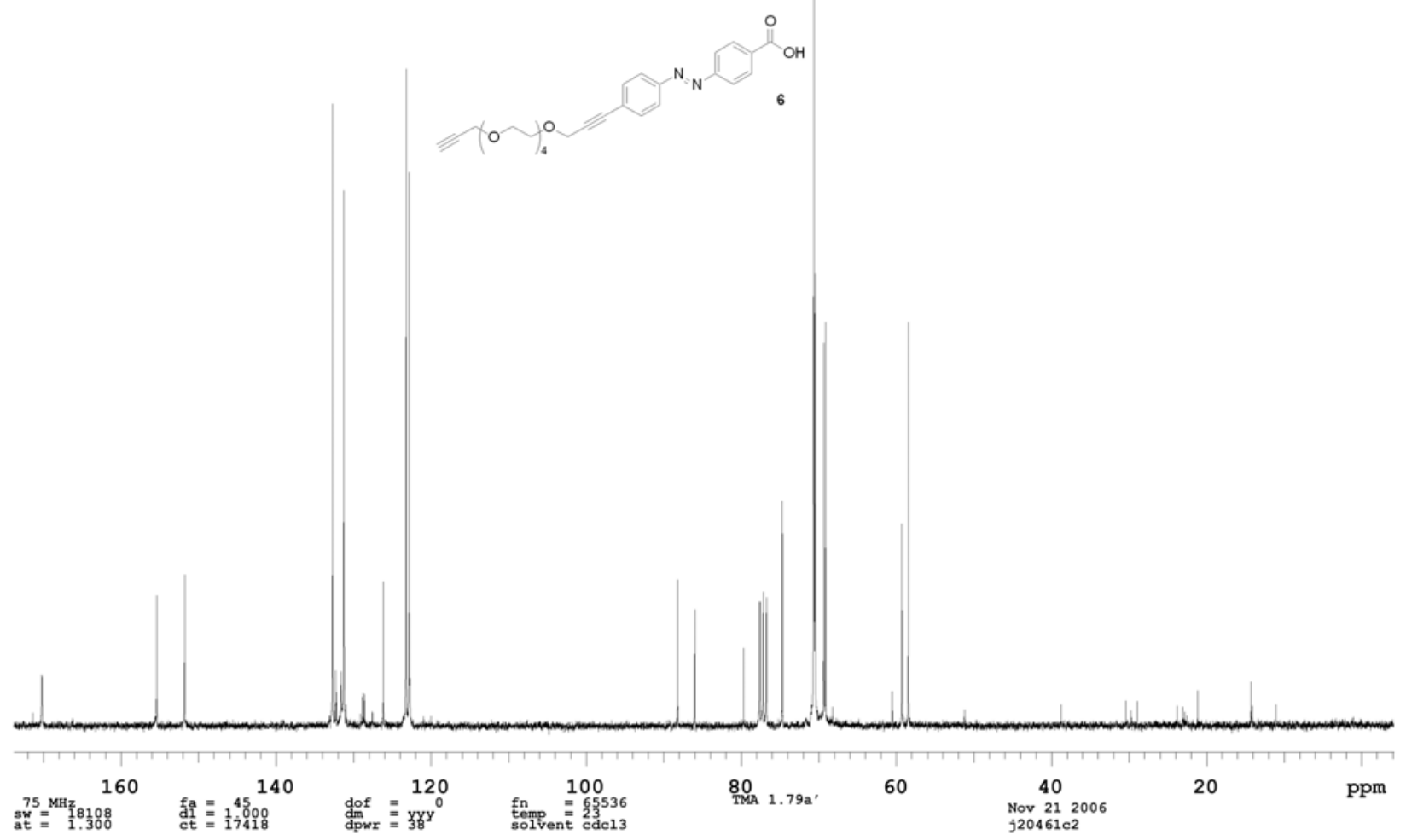




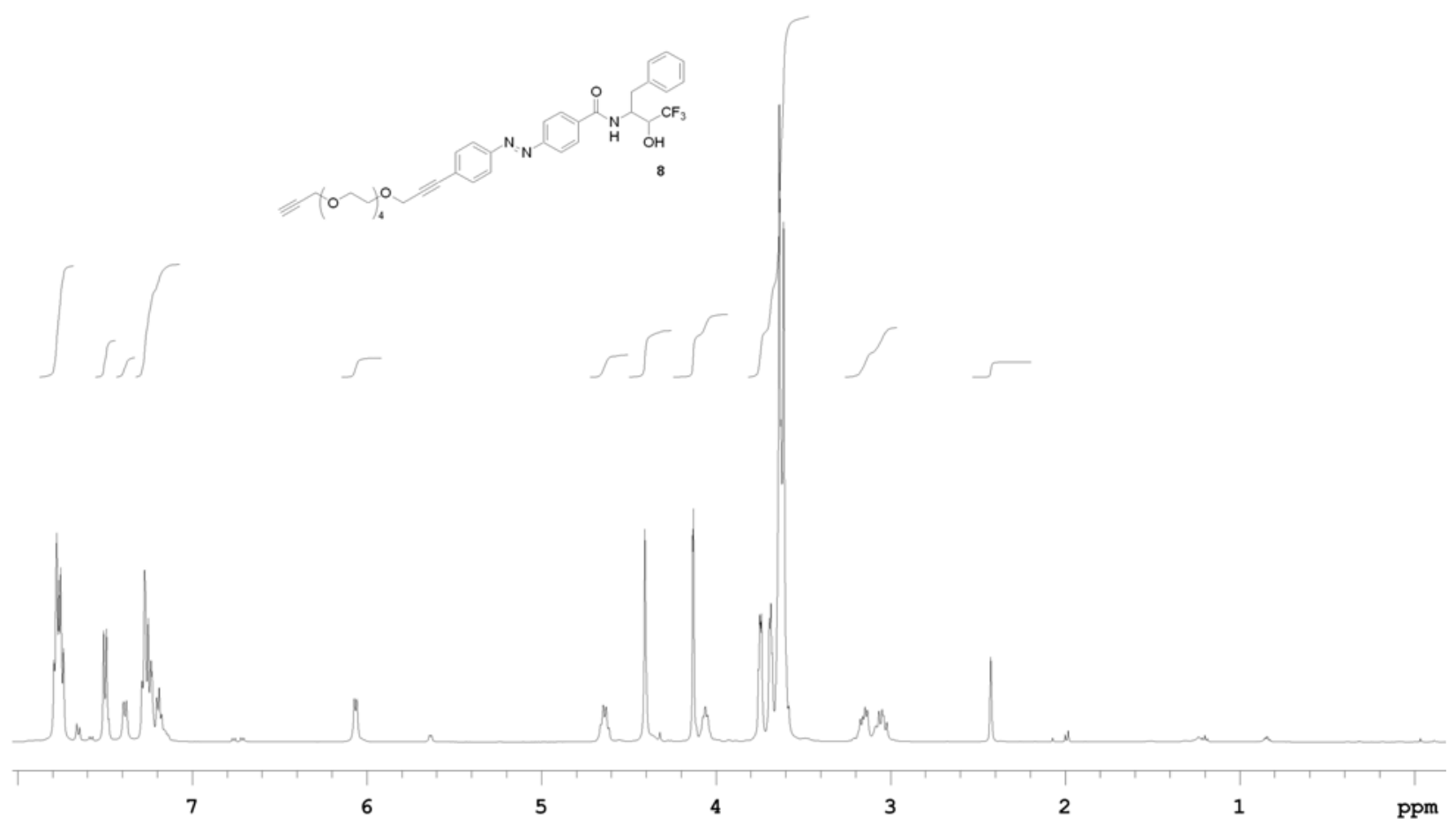

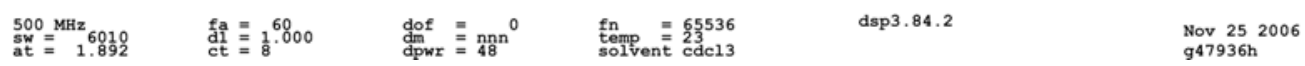

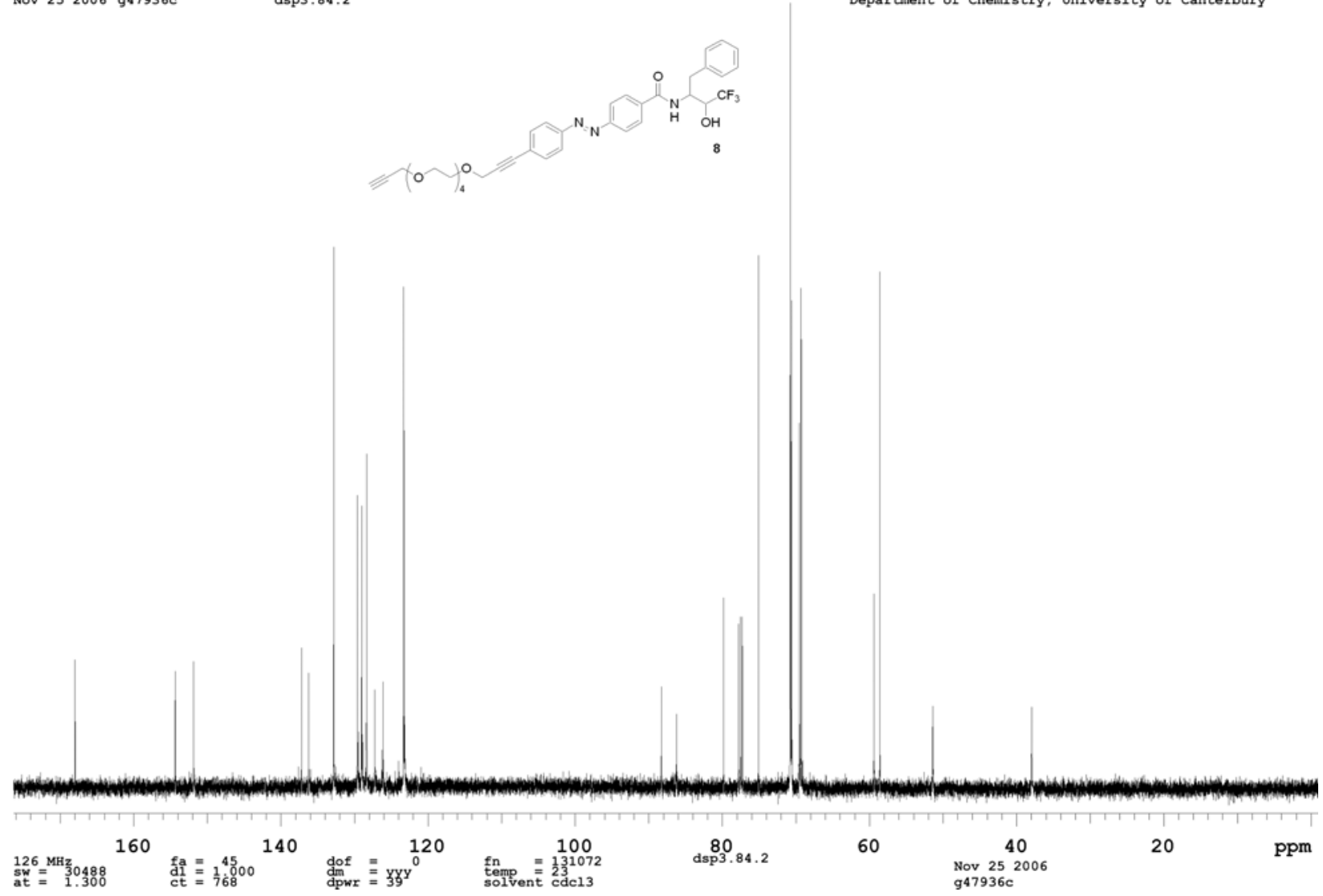




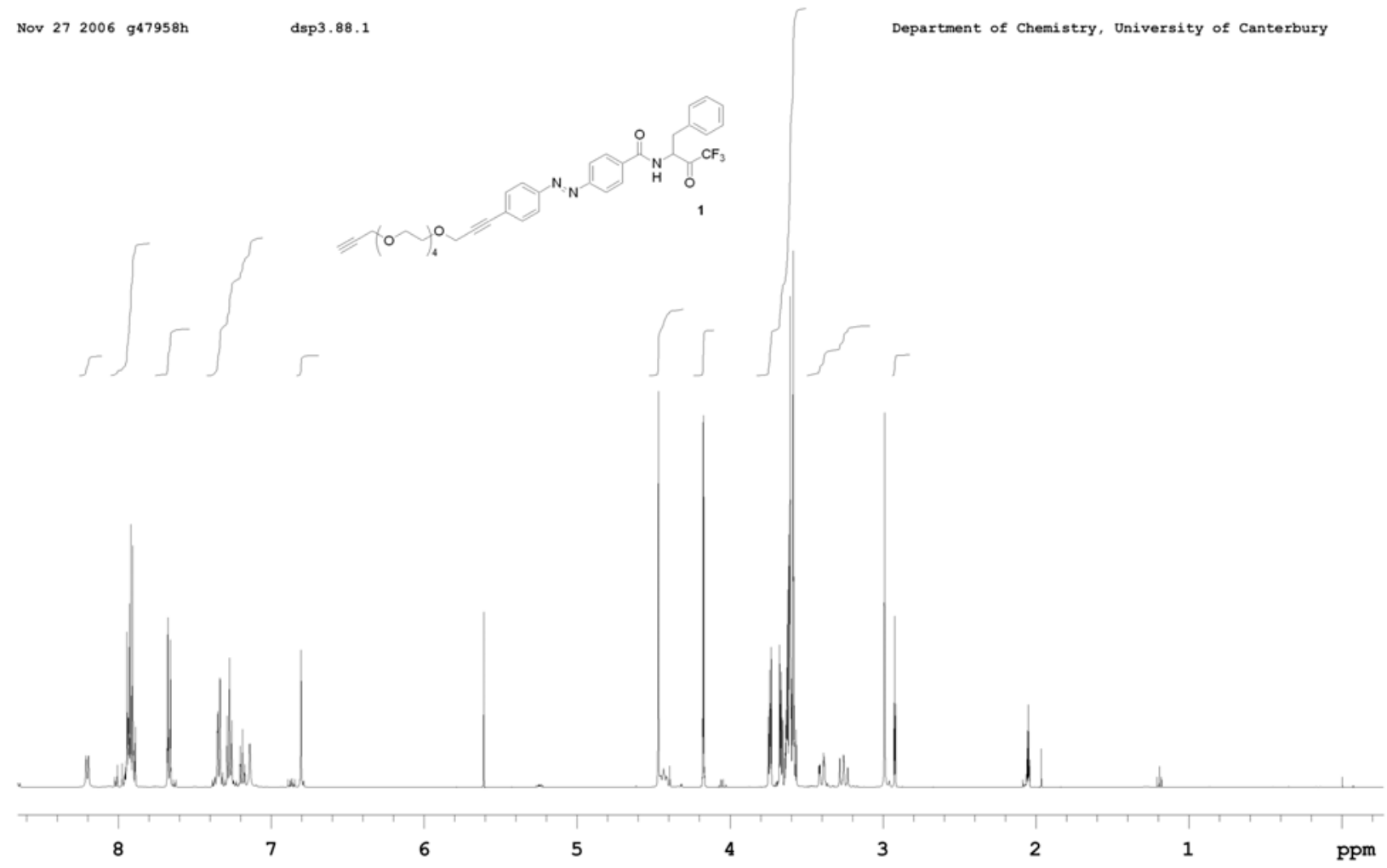

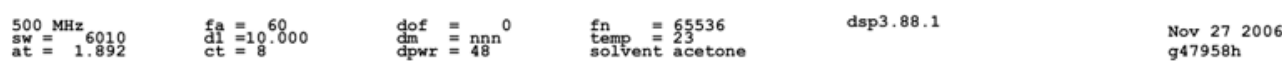

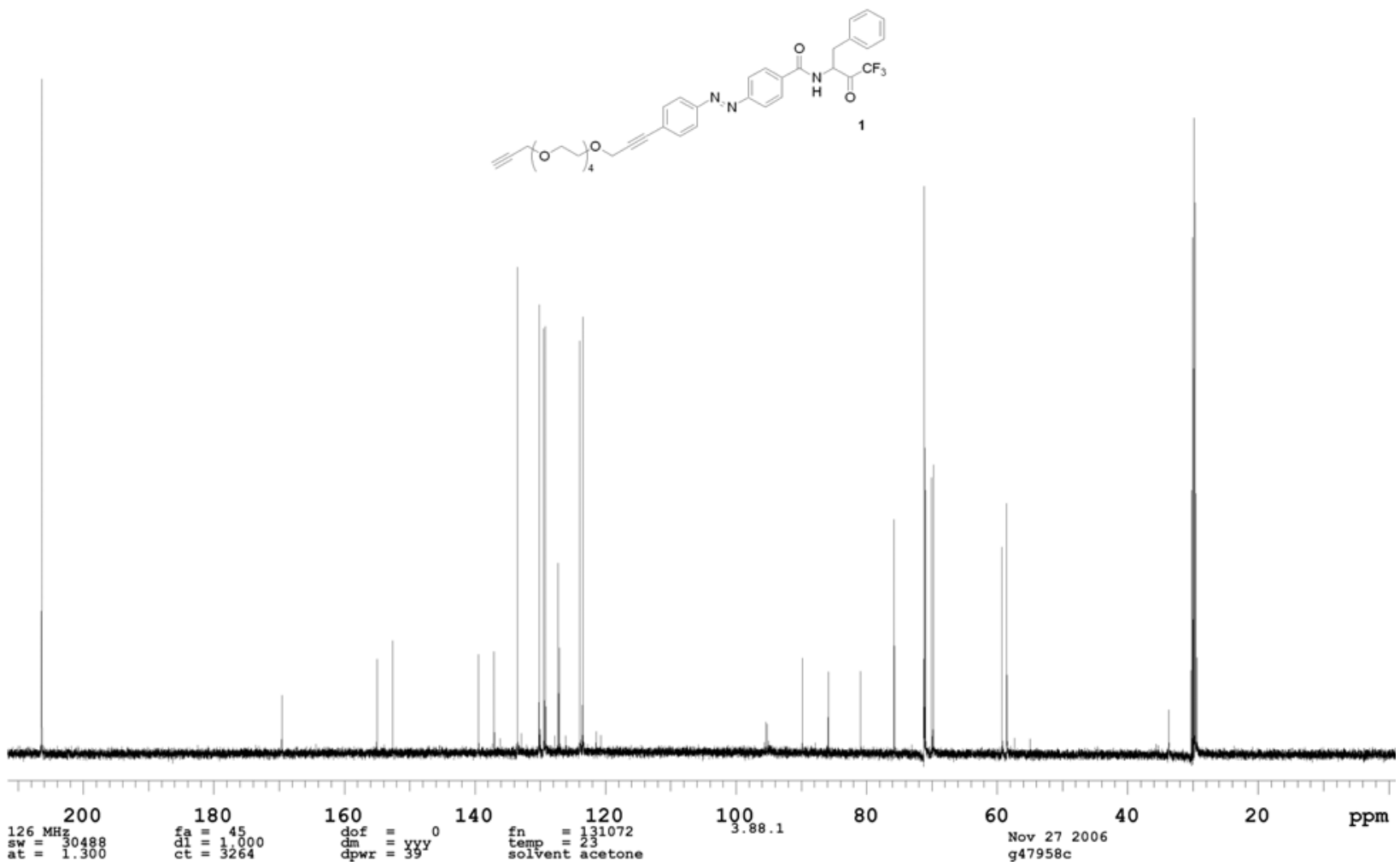




\section{5) References}

1. Pearson, D.; Abell, A. D. Org. Biomol. Chem. 2006, 4, 3618.

2. Westmark, P. R.; Kelly, J. P.; Smith, B. D. J. Am. Chem. Soc. 1993, 115, 3416.

3. Geiger, R., Methods of Enzymatic Analysis. 3 ed.; Verlag Chemie: Weinheim, 1984; Vol. 5, p 99-129.

4. McPhee, M. M.; Kerwin, S. M. Bioorg. Med. Chem. 2001, 9, 2809.

5. Coleman, G. H.; McCloskey, C. M. J. Am. Chem. Soc. 1943, 65, 1588.

6. $\quad$ Peet, N. P.; Burkhart, J. P.; Angelastro, M. R.; Giroux, E. L.; Mehdi, S.; Bey, P.; Kolb, M.; Neises, B.; Schirlin, D. J. Med. Chem. 1990, 33, (1), 394. 